\title{
Concurrent psychiatric comorbidity and multimorbidity in a community study: gender differences and quality of life
}

Abstract This study analysis of a community cohort at the age of 35 focused on the effects of gender and multimorbidity on quality of life and subjective distress. Consistent with an earlier analysis, quality of life decreased with increasing numbers of concurrent psychiatric diagnoses. Women generally reported lower quality of life and higher distress than men. Relative to men, well-being in women was subject to more diagnostic (alcohol abuse/dependence, depression, generalized anxiety disorder, bulimia) and social influences (partner, promotion). The same factors predicted women's psychological and physical well-being, indicating a more holistic experience in women. Men's physical well-being did not correlate with any of the diagnostic or social variables measured.

Key words Quality of life - distress - prevalence - gender $\cdot$ comorbidity $\cdot$ multimorbidity

It was a great pleasure to write this paper in honour of my friend Per Bech, in gratitude for his longlasting friendship and his untiring efforts as President and past-President of the European Association of Psychiatrists and in recognition of his outstanding contribution to research on clinical methodology and quality of life.

\section{Introduction}

Comorbidity or co-occurrence of psychiatric diagnoses has become a important issue in classification: comorbidity is frequent and is associated with greater treatment

Prof. J. Angst (区) - A. Gamma

Zurich University Psychiatric Hospital

Lenggstr. 31, 8029 Zurich, Switzerland

Phone: +4113842611

Fax: +4113842446

E-Mail: jangst@bli.unizh.ch needs, severity and chronicity. Comorbidity denotes primarily the association of two disorders, but many patients receive multiple diagnoses (multimorbidity). This problem has been dealt with mainly in the field of somatic disorders (Gijsen et al. 2001, Xuan et al. 1999). In an earlier analysis we presented data on psychiatric multimorbidity as an indicator of clinical severity on the basis of longitudinal cumulative associations assessed by repeated interviews (Angst et al., submitted). That study showed the association of the number of diagnoses with overall severity, work/social impairment, suicide attempt, treatment and prescribed medication rates, and with distress and quality of life. Associations within diagnostic spectra (for instance between major depression and dysthymia) were more common than between spectra.

This paper presents additional data on the impact of concurrent multimorbidity (1,2 and 3+ diagnoses) on distress and quality of life (QoL) in subjects aged 35 years from the community and gives special consideration to gender differences.

\section{Methods}

The Zurich study is a longitudinal epidemiological investigation carried out in a community sample from the canton of Zurich, Switzerland. In 1978, 4457 young adults (19-20 years old) were screened using the Symptom Checklist 90-Revised (SCL-90R) of Derogatis (1977). For the Zurich study, 591 subjects were selected from this larger sample, with 396 having an SCL-R score above, and 195 below the 85 th percentile. This weighting scheme was used to enrich the sample with potential risk cases. Five interview waves have been conducted so far using the semi-structured SPIKE interview administered by trained clinical psychologists and psychiatrists to assess psychiatric and somatic syndromes, substance abuse, social factors, and quality of life. Symptoms, frequency and duration, degree of distress, treatment history, social 
and work impairment, personal and family history were evaluated for each syndrome. The present analysis is restricted to the participants of the fifth wave conducted in 1993 (215 females and 192 males, aged 34 and 35 respectively).

Psychiatric diagnoses were based on the following criteria: DSM-III: panic disorder, generalized anxiety disorder; DSM-III-R: major depression, dysthymia and bulimia; DSM-IV: bipolar disorder, substance abuse/ dependence; ICD-10: neurasthenia.

The number of psychiatric diagnoses occurring over the previous twelve months was calculated for each subject, considering the following 14 diagnoses: unipolar major depressive disorder $(\mathrm{N}=40)$, unipolar dysthymia (9), bipolar disorder I/II (15), generalized anxiety disorder (37), panic disorder (6), agoraphobia (6), social phobia (6), simple phobia (25), alcohol abuse/dependence (40), drug abuse/dependence (9), benzodiazepine dependence (5), obsessive-compulsive disorder (5), bulimia (4), and neurasthenia (15). Drug abuse/dependence includes abuse/dependence of opiates, hallucinogens, cocaine, amphetamines and cannabis.

Quality of life was evaluated in ten domains: work, finances, family of origin, friends, physical well-being, psychological well-being, spouse/partner, own family, childhood, and total distress. A five-point scale $(5=$ excellent, $4=$ good, $3=$ satisfactory, $2=$ poor, $1=$ very poor) was used to rate quality of life satisfaction or wellbeing in these domains (Bech and Angst 1996). As a further measure, for each of the 14 diagnoses total distress was computed as the sum of the individual distress scores (0-100).

Statistical analyses were carried out with SAS for Windows Version 8.01. Effects of gender and the number of individual diagnoses on the quality of life were assessed non-parametrically with the Kruskal-Wallis Test. Backward multiple linear regression analyses (SAS: PROC REG) were used to compute diagnostic and social predictors of quality of life by gender. All regressions controlled for risk status (stratified sampling of high-risk cases).

Tab. 1 Frequency and prevalence rates as a function of the number of psychiatric diagnoses

\begin{tabular}{lllll}
\hline N of diagnoses & 0 & 1 & 2 & $3+$ \\
\hline N (total = 407) & 271 & 83 & 36 & 17 \\
Women (\%) & 51.3 & 56.6 & 47.2 & 70.6 \\
Prevalence (\%) & 75.4 & 16.9 & 5.0 & 2.7 \\
Women (\%) & 76.5 & 17.9 & 3.1 & 2.5 \\
Men (\%) & 74.2 & 15.4 & 6.9 & 2.9 \\
\hline
\end{tabular}

${ }^{*} p=0.35$
Tab. 2 Mean scores for different domains of quality of life as a function of gender

\begin{tabular}{lrrrrl}
\hline Quality of Life related to: & Men & \multicolumn{4}{c}{ Women } \\
& N & mean & N & mean & \\
& 155 & 3.45 & 197 & 3.25 & .03 \\
\hline Physical well-being & 154 & 3.56 & 197 & 3.26 & .003 \\
Psychological well-being & 156 & 3.47 & 195 & 3.31 & .09 \\
Family of origin (parents, siblings) & 87 & 3.86 & 130 & 3.71 & .06 \\
Own family & 153 & 3.32 & 195 & 3.02 & .005 \\
Childhood/ddolescence & 129 & 3.68 & 169 & 3.31 & .002 \\
Relationship with spouse/partner & 156 & 3.61 & 195 & 3.62 & .99 \\
Relationships in general & 156 & 3.54 & 196 & 3.34 & .02 \\
Work & 156 & 3.37 & 197 & 3.34 & .87 \\
Financial situation & 137 & 74.23 & 177 & 112.54 & .001 \\
Total distress & & & & & \\
\hline
\end{tabular}

* Kruskal-Wallis Test, bold figures are statistically significant

\section{Results}

\section{Frequency and prevalence rates}

As expected, frequency and prevalence decreased with an increasing number of individual diagnoses: 242 subjects of the sample received no psychiatric diagnosis, 83 one diagnosis, 36 two diagnoses and 17 three or more diagnoses (Table 1). Overall there was no gender difference in comorbidity, but females predominated in the group with three or more diagnoses. At the age of 35, the weighted total one-year prevalence of morbidity was $24.6 \%$, with $5 \%$ of the sample receiving two diagnoses and $2.7 \%$ three or more. In contrast to the raw figures the weighted rates demonstrate an equal distribution of coand multimorbidity between males and females.

\section{Quality of life and gender}

Gender-related differences in quality of life ratings are shown in Table 2. Women rated their quality of life consistently lower than men in most domains examined. These differences were statistically significant for physical and psychological well-being, relationship with spouse/partner, work, childhood and total distress; in addition there was a trend $(p<0.1)$ towards a lower quality of life in relationships with family members (both family of origin and own family).

Considering only the subset of subjects without a psychiatric diagnosis (139 females, 132 males) there was a trend towards lower psychological well-being in women (F: mean 3.45; M: mean 3.65; $\mathrm{p}=0.07$ ), but there was no significant gender difference in mean physical well-being scores (F 3.44; $M$ 3.51; $p=0.38$ ). Women without any psychiatric diagnoses rated their QoL in childhood and adolescence much lower than men (F 3.13; M 3.42; $\mathrm{p}=$ $0.02)$. 
Tab. 3 Physical and psychological well-being as a function of gender and the number of psychiatric diagnoses

\begin{tabular}{lllllllllllll}
\hline Nof diagnoses & 0 & & 1 & 2 & \multicolumn{3}{l}{$3+$} \\
\hline well-being & sex & mean SD & mean SD & mean SD & mean SD & $\mathrm{p}^{*}$ \\
\hline physical & $\mathrm{F}$ & 3.44 & 0.74 & 3.05 & 0.93 & 2.87 & 1.06 & 2.45 & 1.13 & 0.001 \\
& $\mathrm{M}$ & 3.52 & 0.77 & 3.23 & 0.86 & 3.33 & 0.99 & 3.00 & 1.0 & 0.39 \\
Fvs M & $\mathrm{p}^{* *}$ & 0.38 & & 0.36 & & 0.20 & & 0.37 & - \\
psychological & $\mathrm{F}$ & 3.45 & 0.89 & 3.07 & 0.91 & 3.00 & 0.93 & 2.09 & 1.04 & 0.001 \\
& $\mathrm{M}$ & 3.65 & 0.75 & 3.42 & 0.70 & 2.92 & 1.08 & 4.00 & 0.00 & 0.01 \\
Fvs M & $\mathrm{p}^{* *}$ & 0.07 & & 0.13 & & 0.72 & & 0.02 & & - \\
\hline
\end{tabular}

* Kruskal-Wallis Test for well-being across different numbers of diagnoses, bold figures are statistically significant;

* Kruskal-Wallis Test for well-being across gender, bold figures are statistically significant; SD standard deviation

\section{Quality of life, multimorbidity and social factors}

Both physical and psychological well-being decreased with an increasing number of concurrent diagnoses (Table 3). In order to analyze the contribution of specific diagnoses and social factors, separate backward multiple linear regression analyses were carried out by gender. Independent predictors were entered into the model as follows: a) social variables: living alone, having a spouse/ partner, number of children, level of training, crucial changes at work, workload, income, regular financial obligations, conflicts at work, promotion, difficulties in relationship with spouse/partner, improvement in relationship with spouse/partner and birth of a child; $b$ ) the psychiatric diagnoses for unipolar major depressive disorder, bipolar disorder I/II , generalized anxiety disorder, simple phobia, alcohol abuse/dependence, bulimia and neurasthenia; (the remaining diagnoses were excluded due to insufficient numbers of subjects), and c) risk status as a consequence of the sample stratification.

In women significant predictors of poorer psychological well-being were alcohol dependence (standardized beta $=-0.28 ; \mathrm{p}<0.001$ ), unipolar depression (beta $=$ $-0.22 ; p=0.003$ ), bulimia (beta $=-0.21 ; p=0.003$ ), difficulties in relationships with spouse/partner (beta $=-0.15$; $p=0.04$ ) whereas promotion exercised a positive effect (beta $=0.20 ; p=0.01$ ). Women's physical well-being was also significantly predicted by bulimia (beta $=-0.24$; $p<0.001$ ), alcohol dependence (beta $=-0.21 ; p=0.005$ ) and difficulties in relationships with spouse/partner (beta $=-0.15 ; \mathrm{p}=0.04$ ) but also by generalized anxiety disorder (beta $=-0.16 ; p=0.02) ;$ promotion again was a positive factor (beta $=0.15 ; p=0.03$ ).

In men the significant predictors of psychological well-being were income (beta $=0.21 ; p=0.01$ ), simple phobia (beta $=-0.19 ; \mathrm{p}=0.02$ ) and sampling stratification (beta $=-0.23 ; p=0.007$ ). Men's physical well-being was not predicted by any of the variables examined.

\section{Discussion}

The large epidemiological surveys (Kessler et al. 1994, Bijl et al. 1998) found no gender differences in the overall lifetime prevalence rates of psychiatric disorders (mood disorders, anxiety disorders and substance abuse/dependence) but reported higher female prevalence rates for comorbid and multimorbid cases. Our one-year prevalence rates for 1,2 and $3+$ diagnoses $(16.9 \%, 5.0 \%$ and $2.7 \%)$ are similar to those reported by Bijl (1998) for the Netherlands ( $15.3 \%, 4.4 \%, 3.8 \%)$. Our analysis of the raw data showed a significant female preponderance among subjects with three or more diagnoses, a difference which disappeared after weighting for the stratified sampling. The relatively small group sizes makes this result inconclusive. In contrast, the National comorbidity survey (Kessler et al. 1994), the Nemesis study (Bijl et al. 1998) and our earlier longitudinal analysis (Angst et al. 2001) all showed consistently higher rates of comorbidity and multimorbidity for females than males.

We found important gender differences in the subjects' rating of their quality of life: women rated their QoL in most domains significantly lower and their distress (as a consequence of psychiatric disorders) significantly higher than men; this result is in agreement with Bech et al.'s (1996) finding that men scored higher on the WHO (Ten) Well-Being Index. Childhood/adolescence received especially low QoL ratings, particularly from women.

The main findings refer to gender differences in psychological and physical well-being, which would not have been expected on the basis of a factor analysis of Ruggeri et al. (2001) where the two loaded on the same factor. Confirming the results of our earlier analysis (Angst et al. 2001), we found that psychological well-being tended to decrease systematically with an increasing number of diagnoses in both men and women. However, for physical well-being this correlation was found only in women.

In the logistic regression, the same social and psychiatric factors predicted women's psychological and physical well-being and the predictive power of those factors was much higher in women than in men. In fact, men's physical well-being did not correlate at all with any of the measures, and their psychological well-being correlated only with two variables (simple phobia and income). These findings can be interpreted as an expression of women's more holistic experience of well-being, whereas men experience physical well-being as independent from psychiatric disorders and social factors.

The obvious limitations of this study are the relatively small sample size and the selection of a cohort at the age of 35. A further limitation is the relatively short instrument, which measures quality of life in 10 domains.

Acknowledgment This work was supported by grant 32-33980-92 of the Swiss National Science Foundation. 


\section{References}

Angst J, Sellaro R, Merikangas KR (2001) Multimorbidity of psychiatric disorders as an indicator of clinical severity. Submitted to Eur Arch Psychiatry Clin Neurosci

Bech P, Angst J (1996) Quality of life in anxiety and social phobia. Int Clin Psychopharmacol 11:97-100

Bech P, Staehr-Johansen K, Gudex C (1996) The WHO (Ten) WellBeing Index: validation in diabetes. Psychother Psychosom 65: 138- 190

Bijl RV, Ravelli A, van Zerssen G (1998) Prevalence of psychiatric disorder in the general population: results of the Netherlands mental health survey and incidence study (NEMESIS). Soc Psychiatry Psychiatr Epidemiol 33: 587-595

Derogatis LR (1977) SCL-90. Administration, Scoring and Procedures Manual-I for the $R$ (revised) version and other instruments of the Psychopathology Rating Scales Series. Johns Hopkins University School of Medicine, Chicago
Gijsen R, Hoeymans N, Schellevis FG, Ruwaard D, Satarino WA, van den Bos GA (2001) Causes and consequences of comorbidity: a review. J Clin Epidemiol 54: 661-674

Kessler RC, Mcgonagle KA, Zhao S, Nelson CB, Hughes M, Eshleman S, Wittchen H-U, Kendler KS (1994) Lifetime and 12-month prevalence of DSM-III-R psychiatric disorders in the United States. Results from the National Comorbidity Survey. Arch Gen Psychiatry 51: 8-19

Ruggeri M, Warner R, Bisoffi G, Fontecedro L (2001) Subjective and objective dimensions of quality of life in psychiatric patients: a factor analytical approach. (The South Verona Outcome Project 4). Br J Psychiatry 178: 268-275

Xuan J, Kirchdoerfer LJ, Boyer JG, Norwood GJ (1999) Effects of comorbidity on health-related quality-of-life scores: an analysis of clinical trial data. Clin Therapeutics 21: 383-403 